\title{
EXPERIMENTAL STUDY OF A CAPACITIVE TOMOGRAPHY SYSTEM FOR MULTIPHASE FLOW
}

\begin{abstract}
L. F. M. Moura, ABSTRACT
E. Cenedese

and A. C. Azevedo Filho

Universidade Estadual de Campinas

Faculdade de Engenharia Mecânica

Departamento de Engenharia Térmica e de

Fluidos

Cidade Universitária "Zeferino Vaz"

Distrito de Barão Geraldo

P.O Box 6122, Campinas, São Paulo, Brasil

felipe@fem.unicamp.br

This paper presents the experimental development of a capacitive tomography system applied to the study of multiphase flows. A capacitance sensor with eight electrodes and a capacitance measurement transducer were constructed. The two-phase flow void fraction was obtained through an electric-mechanical measurement system. The reconstruction of the image of several two-phase flows was obtained using the linear back projection method. Numerical simulation of the capacitance values between electrode pairs were performed, through the method of finite elements, in order to obtain the sensibility maps. This experimental procedure showed the influence of several parameters on the quality of the reconstructed images. The quality of the reconstructed images for air-water and water-oil flows, for different void fractions, demonstrated the validity of the tomography system developed.
\end{abstract}

Keywords: Experimental Study, Tomography, Multiphase Flows, Capacitance Sensor, Linear Back Projection.

\section{NOMENCLATURE}

$\mathrm{B}_{\mathrm{c}} \quad$ calculated liquid fraction

$\mathrm{C}_{\mathrm{b}}$ sum of the capacitances of the electrode pairs

$(1,4)$ and $(5,8)$

$\mathrm{C}_{\mathrm{v}} \quad$ volumetric fraction of dispersed phase

\section{Greek symbols}

$\varepsilon_{1} \quad$ relative permittivity of phase

$\varepsilon_{2} \quad$ relative permittivity of phase

$\varepsilon_{\mathrm{m}} \quad$ relative permittivity of the mixture

$\varepsilon_{0} \quad$ absolute permittivity of vacuum

\section{INTRODUCTION}

The main purpose of tomography is to obtain an image (bidimensional or tridimensional) of an object (parts of the human body, multiphase flow, etc.) through the processing of signals sent and received by sensors located around the object we need to get an image.

Only around 1970 it appeared an image reconstruction technique of the internal organs of the human body, called computed tomography. The image reconstruction techniques in medical tomography had a major boost with the development of the filtered back projection algorithm, by Bracewell and Riddle (1967). Later, Ramanchandran and Lakshinarayanan (1971) also developed the filtered back projection algorithm, where it was applied the convolution theory instead of Fourier transform. According to Kak and Slaney (1988), this algorithm is currently widely used to obtain images in computed tomography.

Around the 80's the tomography was no more restrict only to the medical area and started to have many industrial applications, like in the control and monitoring of industrial processes, known as process tomography or yet as industrial process tomography. Several are the applications of the process tomography in the engineering fields: in the density and flow pattern monitoring in fluidized bed; in volumetric concentration measurements in gas-solid and gas-liquid mixtures; in the measurements of trajectory and velocity of particles. In the petroleum industry, the tomography is applied since the characterization and exploration of reservoirs through the geophysical methods, until the laboratory analysis of rock samples extracted of oil wells, making it easier the study of problems related to viscosity, gravity and mobility control in the gas-water-oil multiphase flows through the rocks.

The use of tomography in industrial processes promoted the appearance of a great variety of tomographic techniques. For industrial applications that aim the monitoring and control of dynamic processes, it was observed that the slow response of the radiation detectors, as well as the slowness of the sequential movements of nucleonic tomography (that uses $\mathrm{X}$ rays or gamma rays), render these techniques indicated only for the obtainment of images of stationary objects, not serving for the objectives of industrial tomography, because it does not possess temporal resolution for such application. To overcome the problems of temporal resolution it 
appears the tomography that uses electrical or magnetic properties. Among them, the electrical capacitance tomography is emphasized because, when compared to the other techniques, it possesses the following advantages: low cost, quick response and it is not dangerous to health like the X-rays and gamma rays. The electrical capacitance tomography can also overcome several problems associated to the application of intrusive sensors. This technique is adequate to applications with components with different relative electrical permittivity and it can be used including for non conductive components.

The first applications of this technique for the measuring of concentration of solid in gas can be found in Morse and Bellou (1951) and Lanneau (1960).

There is a great variety of tomographic techniques that are applied for the image reconstruction both in the medical tomography and in the multiphase processes tomography. All tomographic techniques always have in common the sending and the acquisition of signals from devices that are located all around the object that is imaged. In this paper it is developed an electrical capacitance tomography system for application in multiphase flow industrial processes.

\section{CAPACITIVE TOMOGRAPHY FOR MULTIPHASE FLOW}

The electrical capacitance tomography technique is adequate to processes in which the phases present different electrical permittivities (the phases can be conductors or not conductors). The base of any tomography resides in explore the differences or contrasts in the process's properties. The capacitance sensors are sensible to differences in the electrical permittivity, while the $\mathrm{X}$ rays and the gamma rays are sensible to differences in the density.

The theoretic model relating the electrical impedance or the electrical permittivity of a twophase mixture, with one phase dispersed in a continuous phase, was first presented by Maxwell in 1873. The theory of Maxwell stated that little spheres of a specific material were disseminated in a continuous phase and that the electrical field was disturbed by the presence of the spheres. In its theory, Maxwell assumed the spheres as being of equal size and small compared with the distance between them and formulated the relative permittivity of the mixture according to the equation:

$$
\varepsilon_{\mathrm{m}}=\varepsilon_{1} \frac{2 \varepsilon_{1}+\varepsilon_{2}-2 \mathrm{C}_{\mathrm{v}}\left(\varepsilon_{1}-\varepsilon_{2}\right)}{2 \varepsilon_{1}+\varepsilon_{2}+\mathrm{C}_{\mathrm{v}}\left(\varepsilon_{1}-\varepsilon_{2}\right)}
$$

According to Geldart and Kelsley (1972) and Hage and Werther (1997), the determination of relative permittivity of a multiphase mixture using the concepts of Maxwell is still used today in the monitoring of gas-solid, gas-liquid and liquid-solid systems. Others researchers like Rayleigh (1892), Bottcher (1952) and Meredith and Tobias (1960) also developed works for the calculation of relative permittivity of a mixture of different materials.

The capacitance tomography allows overcoming several problems associated to the application of intrusive sensors and allows a detailed analysis of the morphology of a multiphase flow. The quantity or the arrangement of the phases in the interior of a capacitance sensor may change and the relative permittivity of the medium as well as the capacitance change too. It's these changes the basic idea of the electric capacitance tomography, which is the reconstruction of the spatial distribution a multiphase flow from the capacitance measurements between different pairs of electrodes, associated to some image reconstruction algorithm.

According to Yang and York (1999), the capacitance electric tomography system has the following advantages: non invasive, low cost, quick response, non-health threatening. Still according to these authors, some capacitance tomography systems using sensors with 12 electrodes can collect sufficient data to produce 40 images by second in an online system.

Xiangyuan (2009) presented a new Landweber method for an electrical capacitance tomography system, which was developed to achieve higher speed (online image viewing) and better image reconstruction quality.

Yang (2010) presented an electrical capacitance tomography sensor, where the key issue was the optimization of the sensor, including the number and length of the electrodes, the use of internal and external electrodes surrounding the tube wall, ground wire and guard electrodes.

\section{ALGORITHMS FOR IMAGE RECONSTRUCTION}

For the image reconstruction, from the capacitance measurements, it is necessary the use of mathematical algorithms that allows the reconstitution of the spatial distribution of the relative permittivity of the phases. For this purpose, a diversity of iterative and non iterative algorithms has been created. Some of these algorithms received improvements or were adapted for the tomography process.

The more common algorithms used in image reconstruction for capacitance tomography are: reconstruction based on models, algebraic reconstruction, use of consultation tables, artificial neural networks, simultaneous iterative reconstruction and linear back projection.

The objective of the image reconstruction is to calculate the relative permittivity of each pixel from the all possible capacitance measurements. However, this cannot be done directly because there is no sufficient information. The linear back projection 
method uses a second set of data, known as sensibility map, which may be obtained from numerical simulations for a given sensor. The image is then reconstructed combining the capacitance measurements between the pairs of electrodes with the weighting factor of each pixel (coefficients of the sensibility map.

A tomography system with hard field sensors, like the $\mathrm{X}$ ray sensor, generate a uniform and narrow field in which the sensibility (variation of the measurement as a function of the parameter variation) is independent of the distribution of the parameter inside the sensor. Soft field sensors generate a non-homogeneous field and the sensibility distribution inside the sensor depends on the parameter distribution. In the case of capacitance tomography, a variation in the parameter (relative permittivity) results in a different response depending on the position of this parameter.

According to Xie et al. (1993), Nooralahiyan et al (1993), Mc Kee et al (1993), the linear back projection algorithm is an algorithm typically qualitative and not interactive, being appropriated for the use on real time due to its computerized simplicity.

Jing et al. (2009) presented an algorithm for image reconstruction of electrical capacitance tomography, the standard Tikhonov method, ensuring a high image reconstruction quality. A Newton's method was used for the solution of the objective function. The algorithm was tested with capacitance data without noise and the results indicated that this image reconstruction method is efficient and overcomes the numerical instability of electrical capacitance tomography.

\section{CAPACITANCE MEASUREMENT SYSTEM}

The capacitance measurement system developed in this work consists in a capacitance transducer operating at high frequencies, an optical coupler circuit and a digital multiplexer circuit. The latter allows the computer to sequentially change the electrode pair connected to the data acquisition system. The experimental procedure includes the construction of a capacitance sensor from an acrylic tube around which are fixed eight electrodes. The geometry of the sensor was designed fit the capacitance measurement system, so that the capacitance measurements can produce good quality images.

One of the desirable features of the capacitance transducers used in tomography is that they should be immune to parasitic capacitances. Parasitic capacitances are capacitances that appear in some circuit parts and that influence or alter the true capacitance measurement. These parasitic capacitances are difficult to eliminate due to the variations of the environmental condition (temperature and humidity.
According to Yang et al. (1999), there are three sources of parasitic capacitances in the capacitance tomography measurements: parasitic capacitance between the sensor electrode and the grounded external shield, parasitic capacitance of the coaxial cables that transport the electrical signals $(100 \mathrm{pF}$ per cable meter) and parasitic capacitance of the CMOS keys (around $8 \mathrm{pF}$ ). All these parasitic capacitances, when summed up, can reach values around $150 \mathrm{pF}$. In great part of the industrial applications, it is observed that the capacitance measurements are between 0.01 and $10 \mathrm{pF}$, with capacitance variations of 0.005 to $0.08 \mathrm{pF}$. The parasitic capacitance values are greater than the capacitance variations, showing that the electronic circuits must be designed to be immune to the parasitic capacitances.

For a long time the capacitance transducers are used in applications such as vibration, displacement, pressure and density measurements. In some cases, these capacitance transducers must be able to do dynamic measurements in real time for the use in process control and in other cases are demanded only to be able to do static measurements. For use in process tomography and mainly for the application in multiphase flows, a great variety of electronic circuits have been created.

Huang et al (1992) suggested the following features of a capacitance sensor for the obtaining of images in real time in the oil industry: high resolution ( $0.3 \mathrm{fF}$ ), low noise level ( $\mathrm{rms}$ value lower than 0.1 $\mathrm{fF})$, low variation in the baseline, great amplitude in the measurements $(0.3 \mathrm{fF}$ up to $2 \mathrm{pF})$, high data acquisition rate, switching controlled by computer and data transmission capacity with a distance of up to $250 \mathrm{~m}$.

The performance of the capacitance transducer is very important for the image reconstruction. To reach high sensibility in the measurements, the transducer must possess a high signal/noise ratio and the effects of parasitic capacitances over the measurements must be minimized or eliminated. Therefore, to measure the capacitance accurately, the transducer must: be immune to parasitic capacitances, have high sensibility and stability (low drift) and have low variation in the baseline or must employ methods to reduce this variation, such as selfbalancing and self-calibration.

The two main types of electronic circuits used in electric capacitance tomography are the loading/unloading methods and the AC bridge. The AC bridge circuits are superior to the load/unload circuits relating to low variation in the baseline and high signal/noise ratio. The load/unload circuits have the advantage of operating in different frequencies ( 0.1 up to $5 \mathrm{MHz}$ ). For switching frequencies of up to $2 \mathrm{MHz}$, the load/unload circuits offer a faster data acquisition compared to the $\mathrm{AC}$ bridge circuits. Due to its simplicity, the load/unload circuits allow the use of measurements in parallel, increasing the speed of acquisition without increasing the cost and 
complexity. In the AC bridge circuits, the use of measurements in parallel would imply in a demodulator for each channel, increasing the complexity and cost of the circuit.

\section{DESCRIPTION OF THE EXPERIMENTAL SETUP}

A scheme of the main components of the tomography system used in this work can be seen in figure1.

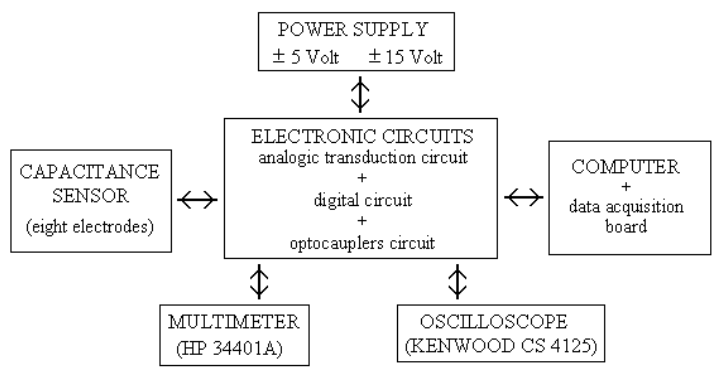

Figure 1. block diagram of the experimental assembly

The capacitance sensor is the component of the tomography system where the capacitance measurements were done. The sensor was built from an acrylic tube with external and internal radius of 30.00 and $26.35 \mathrm{~mm}$, respectively. In the external surface of this tube were placed eight copper plate electrodes with $0.1 \mathrm{~mm}$ thickness, equally separated, with $100 \mathrm{~mm}$ of length, as shown in figure 2. Each one of these electrodes forms a $41^{\circ}$ angle in the center of the sensor and the spacing between two electrodes form a $4^{\circ}$ angle. A detailed view of the sensor with the electrodes can be seen in figure 3. Coaxial cables $(53 \mathrm{ohms}$ ) were welded in the surface of the eight electrodes, encharged to conduct the electric signals. Involving the external part of the sensor, a grounded shield was mounted to protect the circuit from external electromagnetic fields. This shield, made of aluminum plate, is placed at a radial distance of $15 \mathrm{~mm}$ from the electrodes, being supported by two pvc flanges, also covered with aluminum plates and grounded.

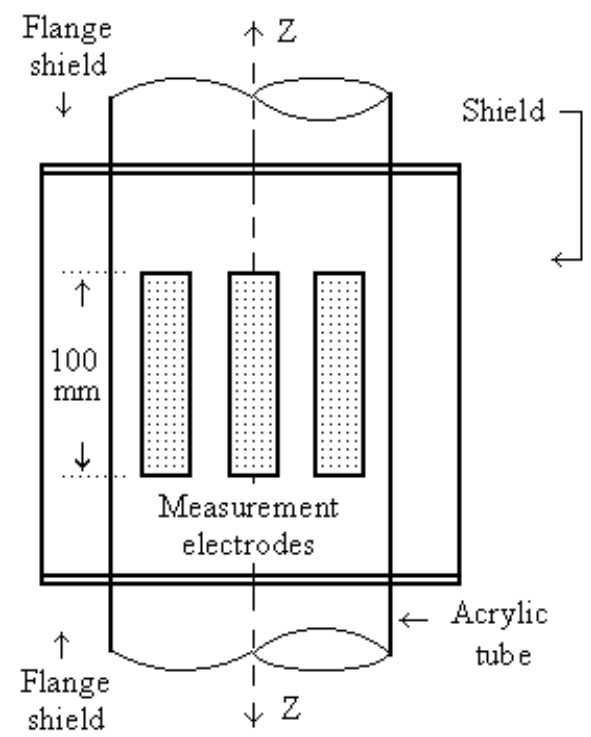

Figure 2. View of the measurement electrodes in the external surface of the sensor

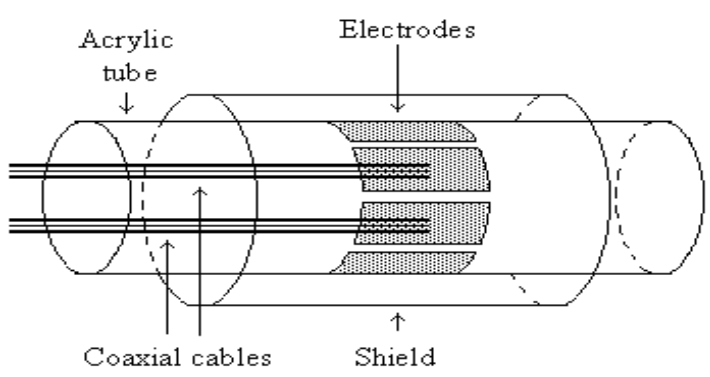

Figure 3. View of the electrodes in the external surface of the sensor, covered by the shield

The electronic circuits are composed of three modules: the capacitance measurement analog circuit, the digital signal multiplexer circuit and the optical coupler circuit that makes the interface with the data acquisition board. A block diagram of these circuits can be seen in Figure 4.

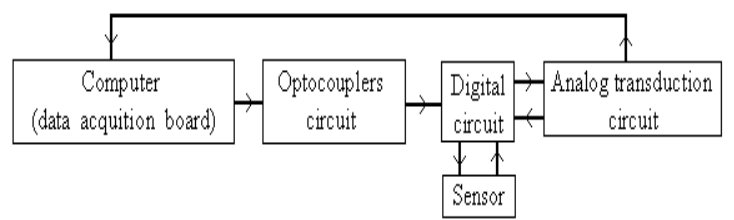

Figure 4. Block diagram of the measurement electronic circuits.

Marioli et al (1993) presented a capacitance measurement technique based on the conversion of AC (proportional to the capacitance) from the sensor electrode in an output signal. This output signal is converted in a DC signal, as shown in the Figure 5. Given that this type of capacitance measurement 
circuit is immune to variations of environmental conditions, the capacitance measurement circuit developed in this work was based on this technique.

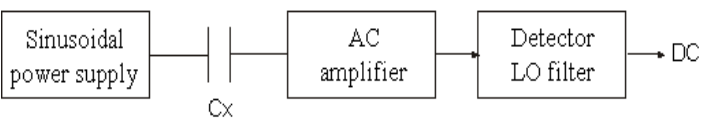

Figure 5. Simplified scheme of the AC circuit

A crystal oscillator provides a sinusoidal signal with frequency of $307 \mathrm{kHz}$ and peak to peak amplitude of $3.5 \mathrm{~V}$. This oscillator possesses relatively high output impedance and requires a current amplifier connected to its output. The signal provided by the oscillator is injected in one of the sensor electrodes and also in the phase detector. The sensor's output current has two components: one displaced $90^{\circ}$ in relation to the sinusoidal signal of the oscillator due to the capacitance and another in phase with the signal due to the conductance. This current is proportionally converted in a voltage signal by an operational amplifier, being later amplified a hundred times by a differential amplifier. The DC component of the signal, proportional to the phase difference between the differential amplifier signal and the reference signal from the oscillator, is separated in a phase detector. It's clear that any signal with angle of lag different of $0^{\circ}$ is associated to the effect of capacitance. The signal passes through an active low-pass filter with Butterworth response and efficiency of $60 \mathrm{~dB}$ around $1.0 \mathrm{kHz}$. Finally, the DC signal is amplified 10 times before the measurement circuit output.

The digital multiplexer circuit is found between the optical couplers circuit and the capacitance transducer, as shown in Figure 4. This circuit is responsible for the sinusoidal signal output to the source electrode and for the voltage signal input from the capacitance transducer. To control the input and output signals were used HCC-4051B multiplexing switches, with eight simple input/output channels. Although this component has eight input/output channels, it was used one component for each source electrode and one for each sensor electrode because this component does not have a good insulation between the input and output channels. In this configuration the HCC-4051B multiplexor presented a leakage signal of less than $1 \mathrm{mV}$ that represents less than $1 \%$ of the lowest measurement. The digital multiplexer circuit used 16 components to control the output sinusoidal signal for the source electrodes and the input signals coming from the sensor electrodes. The connection of all electronic set with the sensor is made through the digital multiplexer circuit, by means of BNC type terminals where coaxial cables are connected.

The interface between the data acquisition board and the digital multiplexer circuit is made by the optical couplers circuit. This circuit was built with the purpose to protect the data acquisition board against short circuit and is composed of two parts: one connected to the source electrodes digital circuit and another connected to the sensor electrodes digital circuit. The optical couplers circuit receives the pulses from the computer and connect the output sinusoidal signal to the digital multiplexer circuit, which control the output to the source electrode and the signal input from the sensor electrode.

The data acquisition system was formed by a microcomputer and a LYNX - CAD 12/32 data acquisition board. This board has 16 analog input channels, 16 digital input channels, 16 digital output channels, one 12 bit analog-digital converter and a converting time of $32 \mu \mathrm{s}$. It was used an analog input channel for the voltage reading from the capacitance transducer. The electrode pairs multiplexing involved in each measurement was made by a program that also controls several parameters of the data acquisition system, like: rate and speed of the data acquisition, quantity of measurements for each electrode pair and average values of the measurements.

To measure the void fraction in the stratified two-phase flow pattern it was used a level detection technique A depth micrometer, associated to an electric circuit, detects when the needle coupled in the micrometer pole touches the water. The micrometer is placed in an adapter positioned in the superior part of the sensor, performing a vertical movement until it touches the air-water interface. The height of the water in the interior of the sensor was determined by reading the displacement of the micrometer's rod.

\section{CALIBRATION OF THE CAPACITANCE MEASUREMENT CIRCUIT}

To calibrate the capacitance measurement circuit it was used a capacitor bank composed of 16 NPO type ceramic capacitors (with $1 \%$ of precision), calibrated with precision equipment. A metal box was constructed with the capacitors displayed inside, so that they don't suffer any interference of the external electromagnetic fields neither the influence of parasitic capacitances. The connection of the metal box with the other equipment was made through two coaxial cables with BNC type terminals.

To ensure reliability of the capacitance values, the capacitor bank was calibrated using a HP4284A LCR bridge meter (precision of $\pm 0,005 \%$ at 307 $\mathrm{kHz}$ ).

Before the calibration of the capacitance transducer, it was verified the circuit stabilization time (warm-up). To achieve these tests, all electronic circuits were turned on and the $1.88131 \mathrm{pF}$ capacitor was connected to the BNC terminals. The potentiometer connected to the amplifier of the measurement circuit was adjusted to provide an output signal of $0.87047 \mathrm{~V}$. Then were performed 15 
output measurements, during a period of 45 minutes. It was observed that after 20 minutes the output value stabilized near an average value.

To establish the stability of the circuits, it was verified the variation of the baseline in relation to time (drift). After the end of the warm-up period, the potentiometer connected to the amplifier of the measurement circuit was adjusted to provide an output signal of $1.00053 \mathrm{~V}$. After 8 hours it was performed another reading of the output signal, resulting in a drift of $0.00162 \mathrm{~V}$ (near $0.1 \%$ ).

For the calibration of the capacitance meter, the $1.88131 \mathrm{pF}$ capacitor was connected to the meter and the potentiometer of the amplifier was adjusted to provide an output signal of $0.96490 \mathrm{~V}$. Thereafter the output signal was read for each one of the capacitors (32 measurements were made and an arithmetic average was established). The output signal average values, for the capacitors from $0.36671 \mathrm{pF}$ up to $7.52887 \mathrm{pF}$, were used in the calibration procedure, presented in Figure 6. It can be observed in this figure a linear behavior.

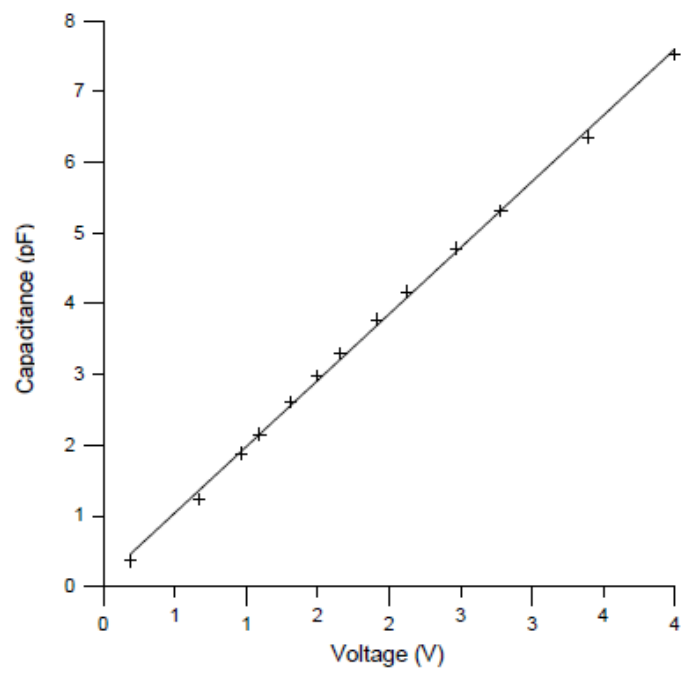

Figure 6. Capacitance meter calibration curve

The equation that represents the capacitance meter calibration curve, obtained through a linear regression (determination coefficient equal to $0.9987)$, is given by:

$\mathrm{C}=1.87396 \cdot \mathrm{V}+0.107479$

The uncertainty of the capacitance values obtained through the capacitance meter calibration curve was calculated using the $t$ distribution of Student, for a confidence interval of $95 \%$. This uncertainty is a composition of the uncertainty resulted from the capacitor bank calibration (systematic error) associated with the uncertainty resulted from the linear regression (random error).
The systematic error in the capacitor bank calibration is given by the precision of the calibration device (HP4284A) which is \pm 0.0005 of the measured value. The $t$ of Student for 4 samples has the value of 2.78 and the biggest deviation of all samples is equal to $1.06552 \mathrm{E}-3$. Thus, we obtain an uncertainty for the capacitor values of $\pm 0.00315 \mathrm{pF}$.

The uncertainty in the values of capacitances obtained through the calibration curve was calculated from the number of points in the curve (equal to 13), the regression polynomial degree (equal to 1), the adjustment curve standard deviation values, the confidence interval (equal to 95\%), the $t$ value of Student (equals to 2.16) and the uncertainty of capacitance values of the capacitor bank. From these data, it was obtained an uncertainty in the capacitance values, obtained through the calibration curve, of \pm $0.03115 \mathrm{pF}$.

\section{CAPACITANCE MEASUREMENTS}

The experimental tests were performed for airwater flow (stratified flow pattern) and oil-water (concentric and eccentric annular flow patterns), for several void fraction values. For each void fraction value, the average values of 30 capacitance measurements were obtained for each one of the 28 electrode pairs. This data set was necessary for the flow image reconstruction. During multiplexing, it was defined a time for the signal stabilization of 30 $\mathrm{ms}$ between the change of the source electrodes and a time of $25 \mathrm{~ms}$ between the changes of the sensor electrodes.

Initially measurements were done with the sensor totally filled with air, with water and with oil. Table 1 presents the capacitance measurements for these cases.

Table1. Capacitance measurements for the sensor filled with air, water and oil.

\begin{tabular}{|c|c|c|c|}
\hline \multirow{2}{*}{$\begin{array}{c}\text { Pair of } \\
\text { electrodes }\end{array}$} & \multicolumn{3}{|c|}{ Capacitance $(\mathrm{pF})$} \\
\cline { 2 - 4 } & air & oil & water \\
\hline$(1,2)$ & 2.17803 & 3.46407 & 4.98533 \\
\hline$(1,3)$ & 0.48453 & 0.84175 & 3.95337 \\
\hline$(1,4)$ & 0.39917 & 0.65510 & 3.90891 \\
\hline$(1,5)$ & 0.35806 & 0.63271 & 3.86539 \\
\hline
\end{tabular}

For the case of air-water stratified flow, it was found a relation between the capacitance measurements (for the 28 electrode pair combinations) and the liquid fraction (ratio between the area occupied by the liquid and the internal area of the sensor), for 16 different values of the liquid fraction, varying from 5.20 to $90.59 \%$. The liquid fraction is necessary as input data for the image reconstruction algorithm.

Analyzing the behavior of the liquid fraction as a function of the capacitance measurements for each one of the 28 electrode pairs it is observed that, using capacitance values of some electrode pair 
combinations, an almost linear relation can be obtained. After testing several combinations, the better combination was the electrode pairs $(1,4)$ and $(5,8)$. A very good regression for this relation was obtained by the polynomial represented by equation (3). The behavior of the regression of the liquid fraction as a function of the capacitance measurements of the electrode pairs $(1,4)$ and $(5,8)$ is illustrated in Figure 7. It is worth to remember that this equation is valid only for air-water stratified flow, because according to Auracher et al. (1985) the capacitance is sensible both to the permittivity distribution of and the flow pattern.

$$
\begin{aligned}
& \beta_{\mathrm{c}}=28,9861-1666 \cdot \mathrm{C}_{\mathrm{b}}+375815 \cdot \mathrm{C}_{\mathrm{b}}{ }^{2}- \\
& -351163 \cdot \mathrm{C}_{\mathrm{b}}{ }^{3}+163063 \cdot \mathrm{C}_{\mathrm{b}}{ }^{4}-36,3898 \mathrm{C}_{\mathrm{b}}{ }^{5}+ \\
& +3,11902 \cdot \mathrm{C}_{\mathrm{b}}{ }^{6}
\end{aligned}
$$

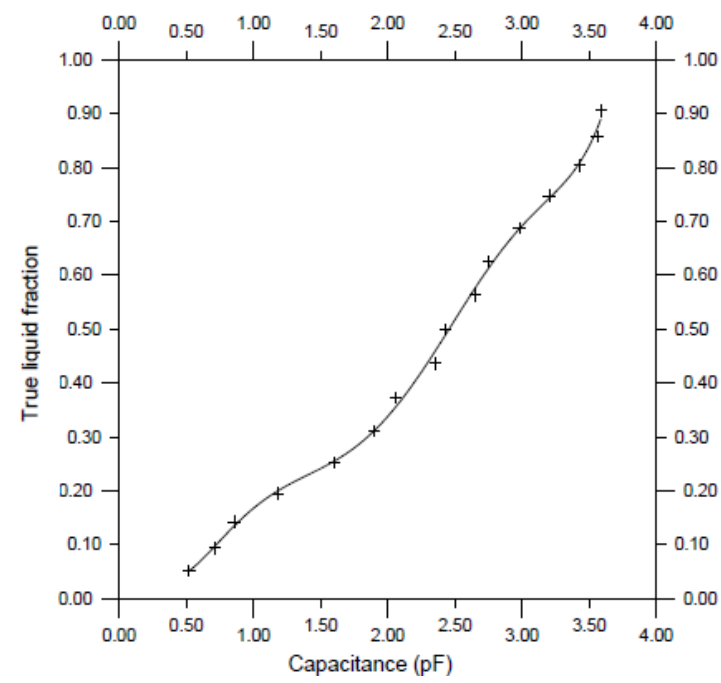

Figure 7. Relation between the liquid fraction and the capacitances

The uncertainty in the liquid fraction values, obtained through the above regression curve, involve the systematic error resulted from the void fraction measuring equipment (micrometer) and the random error of regression. From the number of points in the regression curve (equal to 16), the degree of the polynomial (equal to 6), the curve standard deviation value (equal to 0.0131) and the confidence interval of 95\% ( $t$ value of Student equal to 2.2), the uncertainty in the liquid fraction values obtained through the regression is $\pm 0,0298$.

\section{RESULTS FOR AIR-WATER STRATIFIED FLOW}

To obtain an image (the degree of grey of each pixel) of a multiphase flow, for a given void fraction value, the following input files are needed: the sensibility map of the sensor for the multiphase mixture, the capacitance measurements between the electrode pairs and the liquid fraction. The liquid fraction is necessary for the cut-off level resource in the linear back projection algorithm for the reconstruction of sharper images. The detailed description of the linear back projection algorithm for the image reconstruction with the cut-off level resource can be found in Azevedo Filho (2002).

The capacitance measurements for the stratified air-water flow were done for the following void fraction values (in \%): 5,20; 9,41; 14,24; 19,55; 25,$23 ; 31,19 ; 37,35 ; 43,64 ; 50,00 ; 56,35 ; 62,65$; 68,$81 ; 74,77 ; 80,45 ; 85,76 ; 90,59$ and 94,80 .

Figures 8 to 13 show the surface images (tridimensional representation) and the interface image (transversal section of the sensor) for some void fraction values. The surface images of this flow pattern were presented with a rotation to make the visualization of the interface easier. Two interface images are presented for each void fraction value: the real image (or experimental) and the reconstructed image. It can be observed a great agreement between the reconstructed image and the real image, proving the validity of the adopted methodology.

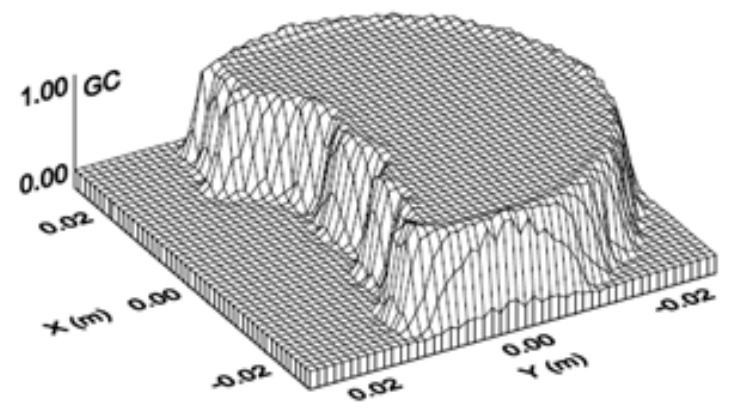

Figure 8. Surface image of the air-water stratified flow $(25.23 \%$ void fraction

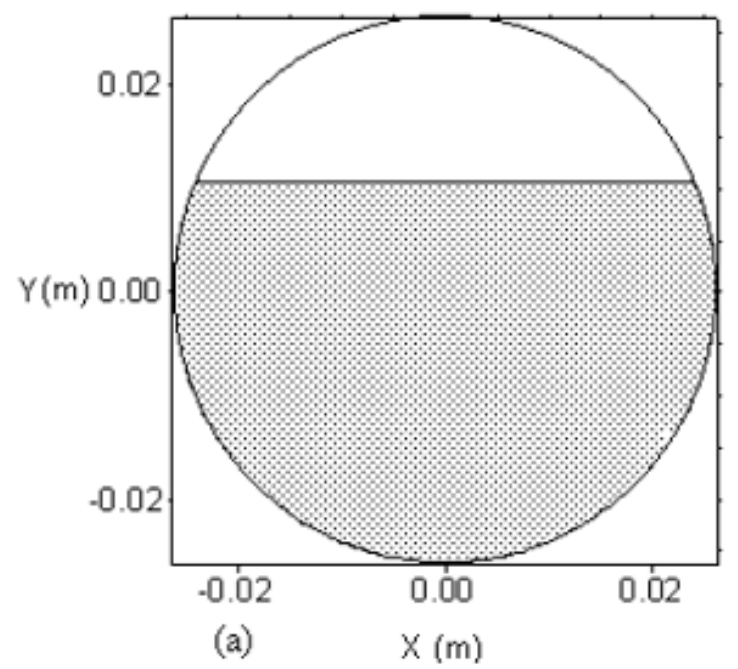

(a)
$X(\mathrm{~m})$ 


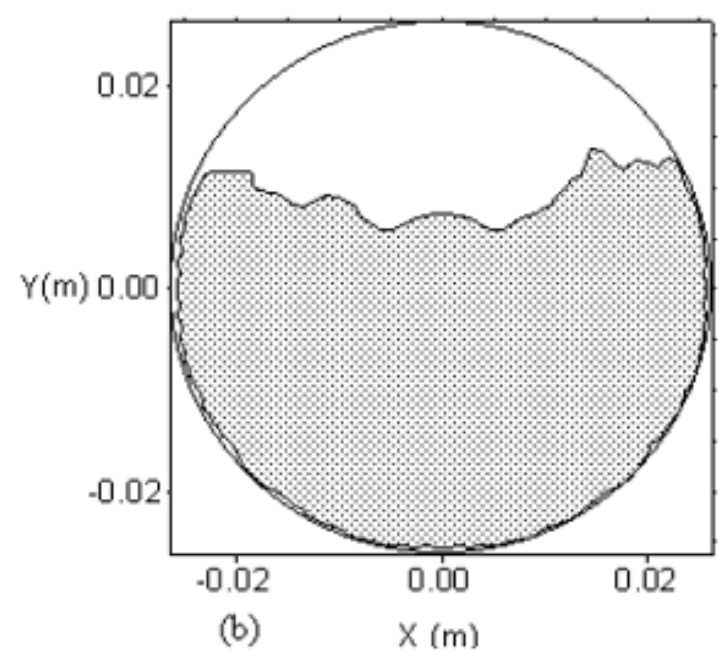

Figure 9. Images (a) experimental and (b) reconstructed of the air-water stratified flow $(25.23 \%$ void fraction

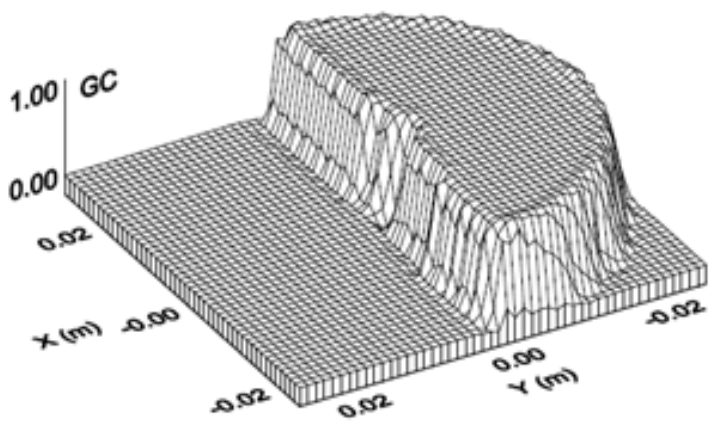

Figure 10. Surface image of the air-water stratified flow $(50.00 \%$ void fraction

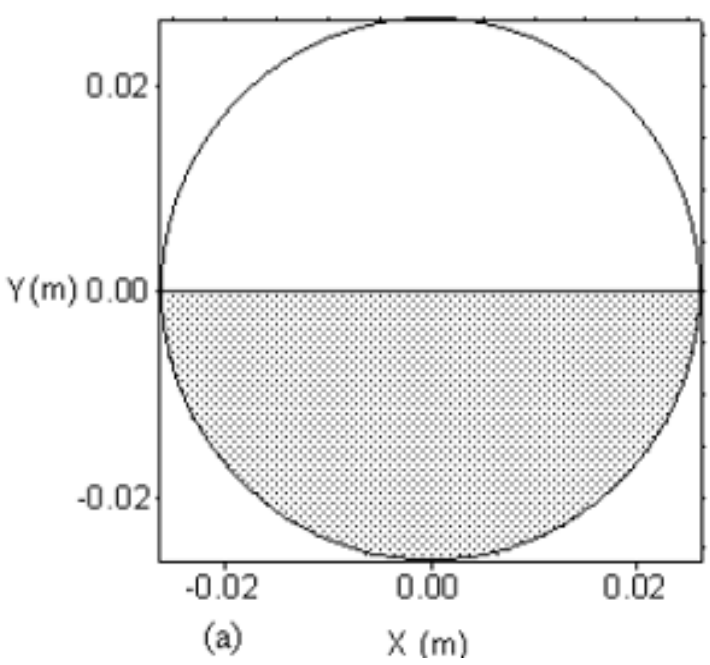

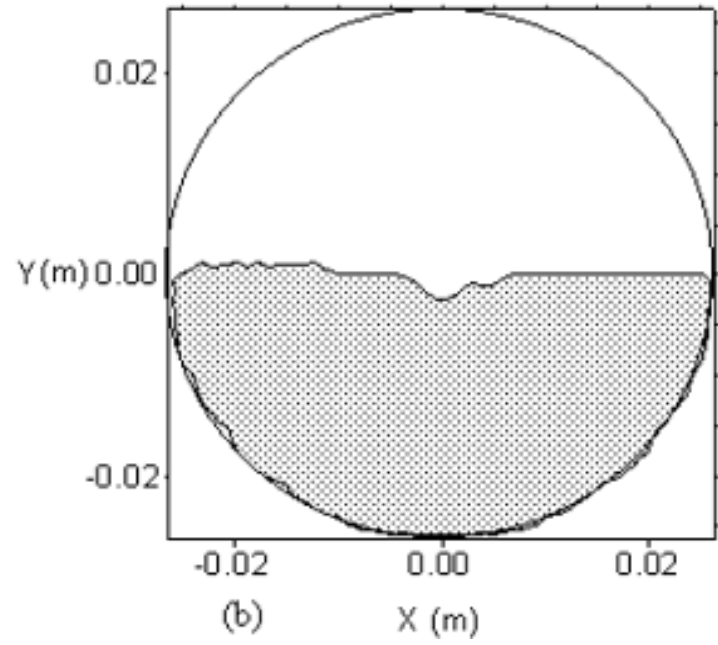

Figure 11. Images (a) experimental and (b) reconstructed of the air-water stratified flow $(50.00 \%$ void fraction)

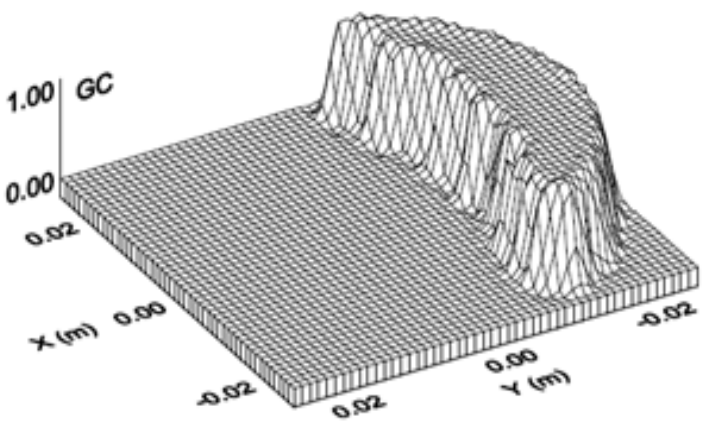

Figure 12. Surface image of the air-water stratified flow (74.77\% void fraction)

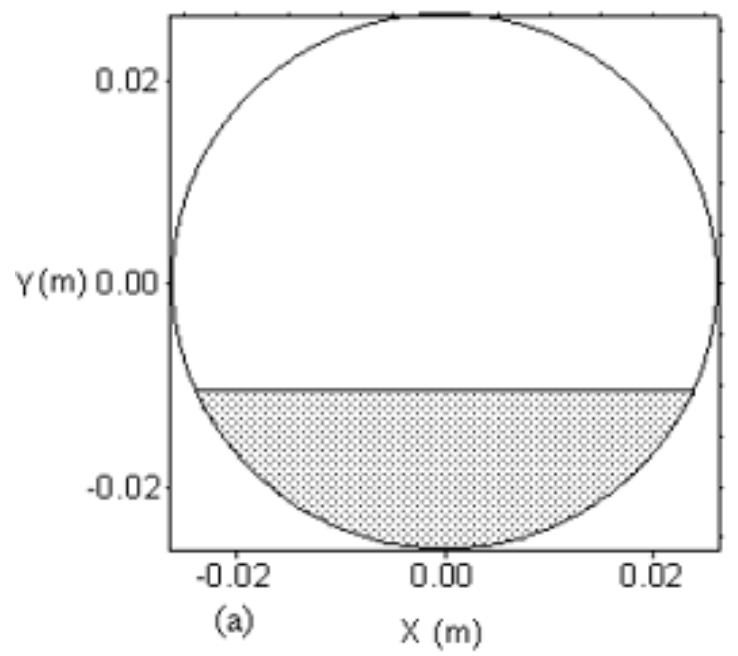




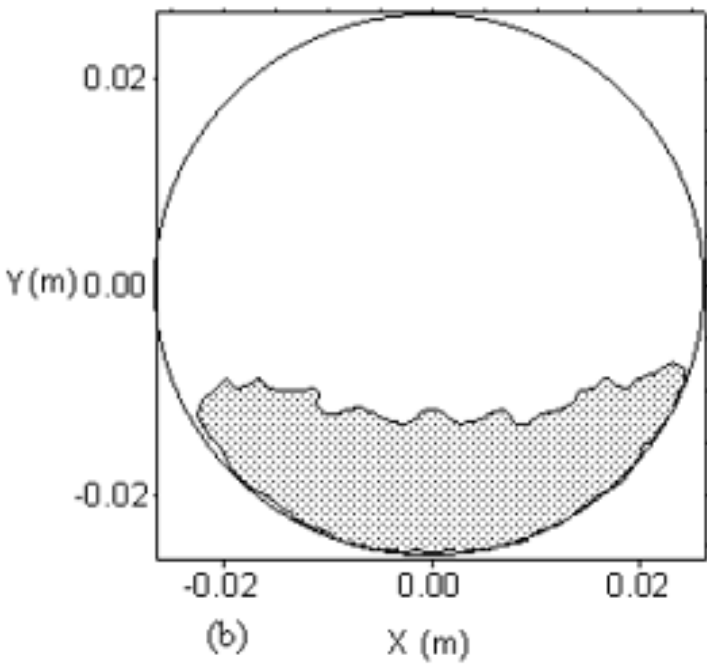

Figure 13. Images (a) experimental and (b) reconstructed of the air-water stratified flow $(74.77 \%$ void fraction)

\section{RESULTS FOR THE WATER-OIL ANNULAR FLOW}

The water-oil annular flows were simulated inserting a nylon cylinder (the same relative permittivity of the oil) in the interior of the sensor filled with water. The eccentricity of the oil core was simulated moving the nylon cylinder in the vertical direction. Water-oil concentric annular flow (void fraction equals to $19.05 \%$ ) and water-oil eccentric annular flows (void fraction equal to 32.41, 44.11, and $57.61 \%$ ) were simulated. The void fraction in the water-oil flow is defined as the ratio between the area occupied by oil and the internal area of the sensor.

Due to the low quantity of experimental data, it was not possible to obtain a relation between the capacitance measurements and the liquid fraction. Therefore, the liquid fraction value used as input for the image reconstruction algorithm was the liquid fraction obtained through the relation $\beta=1-\alpha$

Figures 14 to 19 showed the surface images (tridimensional representation) and the interface image (transversal section of the sensor) for some void fraction values. The surface images of this flow pattern were presented with some rotation to make the visualization of the interface easier. The interface images, in the case of the eccentric annular pattern, were reversed. Two interface images are presented for each void fraction: the real image (or experimental) and the reconstructed image. It can be observed again a good concordance between the reconstructed image and the real image, proving the validity of the adopted methodology.

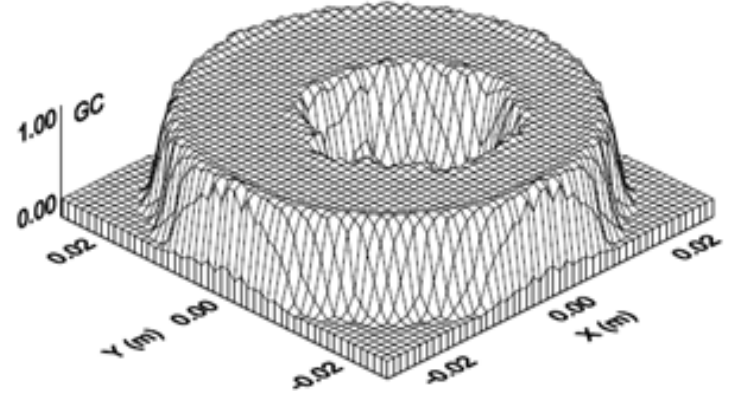

Figure 14. Surface image of the water-oil concentric annular flow (19.05\% void fraction)
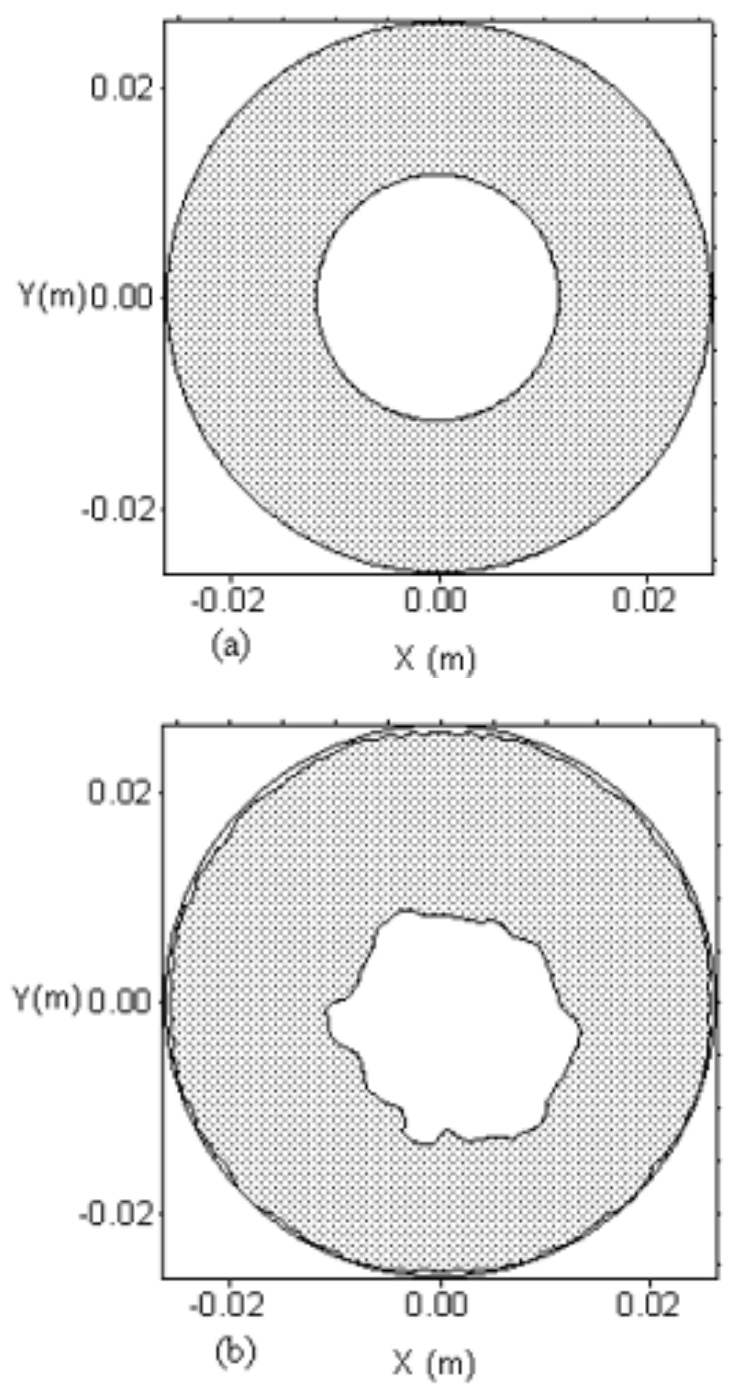

Figure 15. Images (a) experimental and (b) reconstructed of the water-oil concentric annular flow (19.05\% void fraction) 


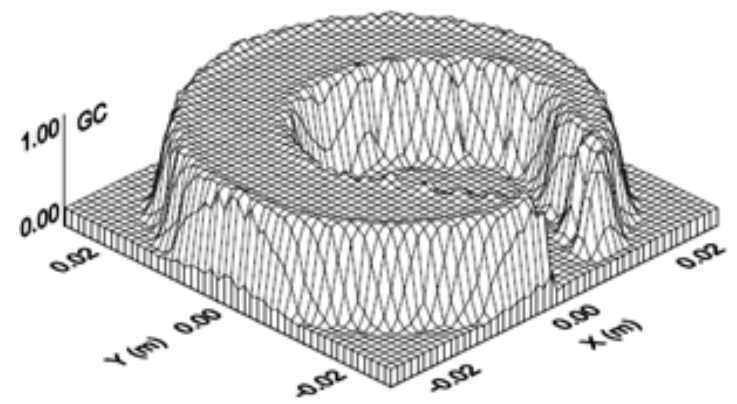

Figure 16. Surface image of the water-oil eccentric annular flow (32.41\% void fraction)
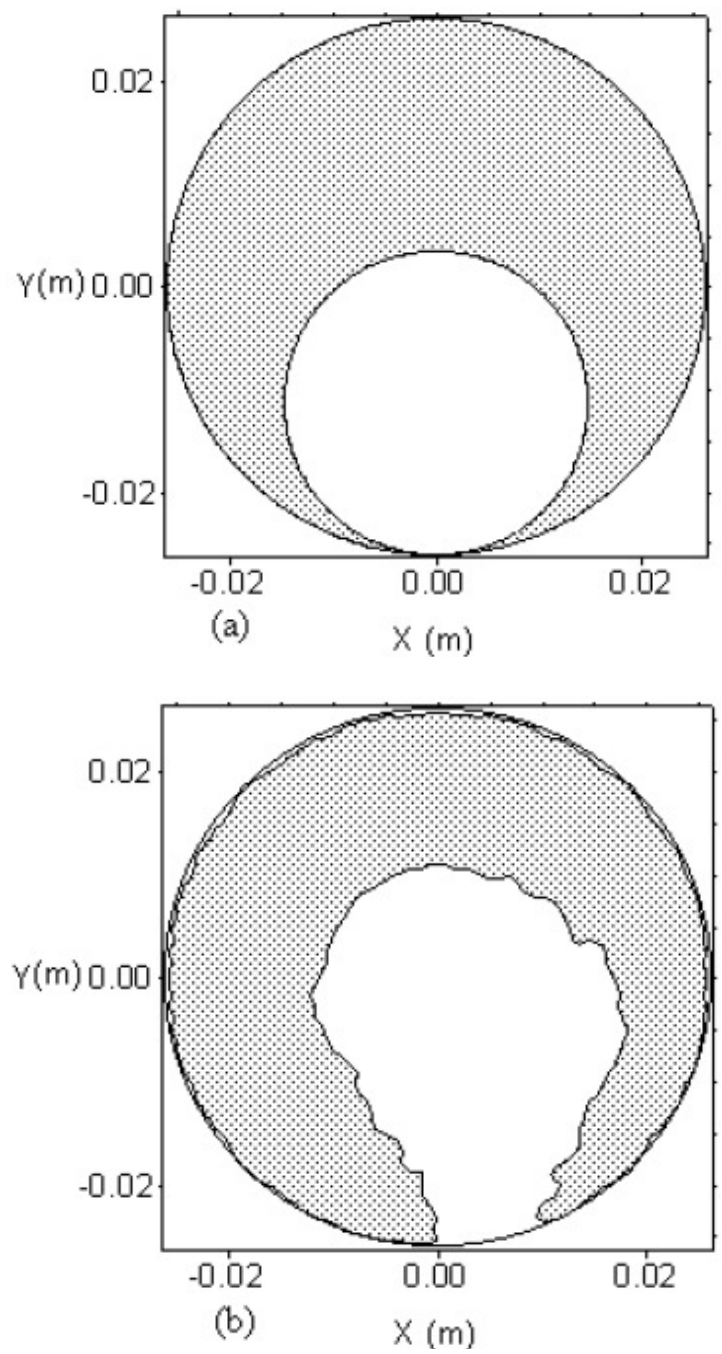

Figure 17. Images (a) experimental and (b) reconstructed of the water-oil eccentric annular flow (32.41\% void fraction)

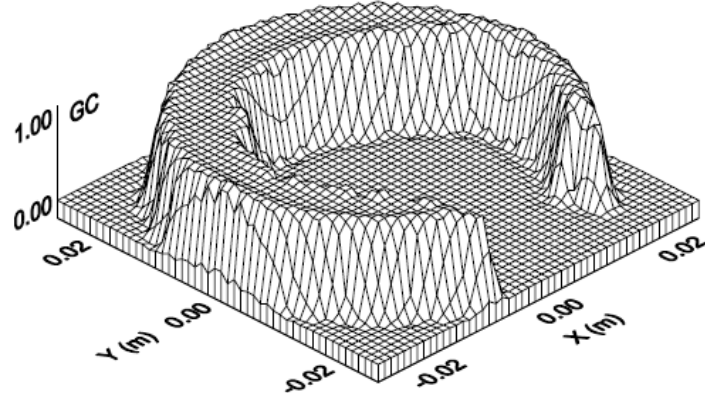

Figure 18. Surface image of the water-oil eccentric annular flow $(57.61 \%$ void fraction
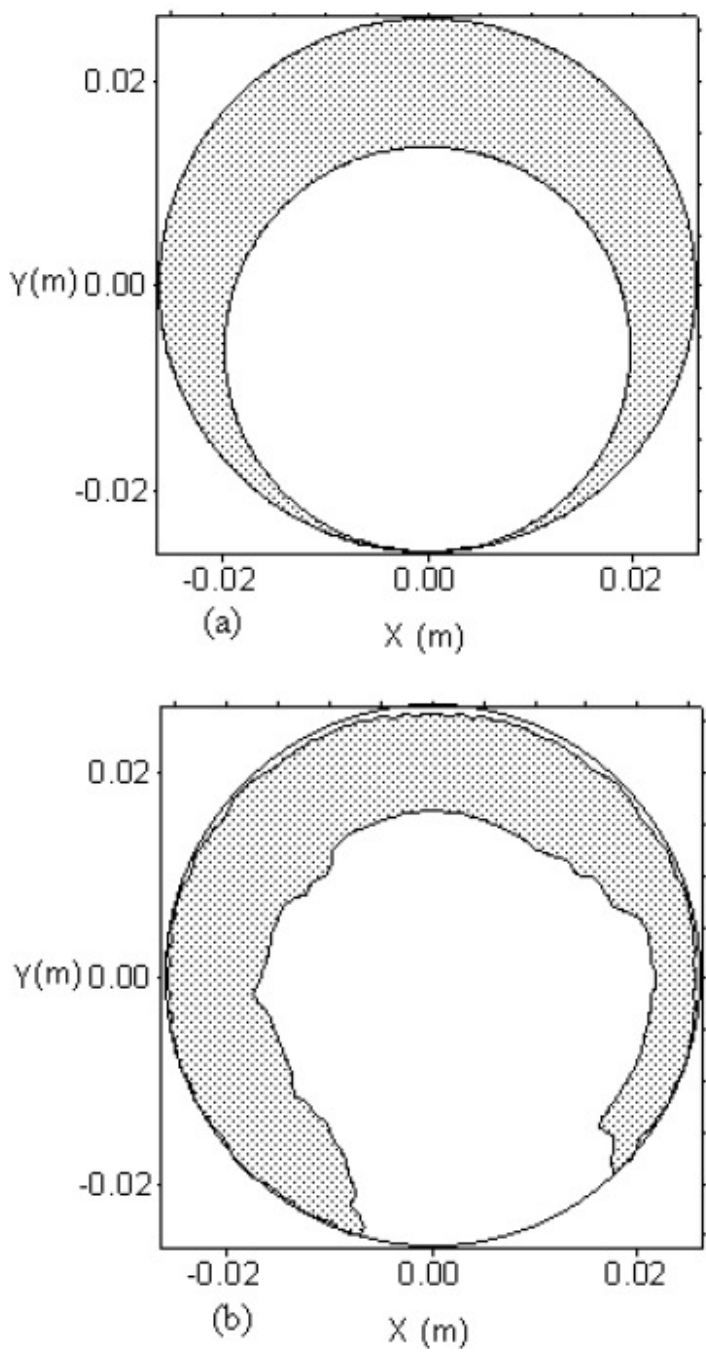

Figure 19. Images (a) experimental and (b) reconstructed of the water-oil eccentric annular flow (57.61\% void fraction)

\section{CONCLUSIONS}

This paper presents the experimental development of a capacitive tomography system applied to the study of multiphase flows. The 
tomography system consists in a capacitance sensor with eight electrodes placed at the external surface of an acrylic tube and three electronic circuits: a capacitance meter, a multiplexing circuit and an optical couplers circuit, besides the data acquisition system.

For the calibration of the capacitance meter it was used a bank of 16 capacitors, calibrated with a precision device. The uncertainty involved in the capacitance values of this bank was of \pm 0.00315 pf. In the calibration procedure of the capacitance meter it was observed that the circuit is almost linear, what was proved by a regression coefficient of determination equals to 0.9987 . The statistical analysis of the capacitance values obtained through the calibration curve showed that the uncertainty in the capacitance measurements was $\pm 0.0312 \mathrm{pf}$.

Experimental tests for air-water stratified flow and water-oil concentric and eccentric annular flows were performed. For air-water stratified flow, the tests were performed for 17 different values of liquid fraction, in order to obtain a relation between the liquid fraction and the capacitance measurements. The uncertainty in the obtainment of the liquid fraction through this relation was only \pm 0.029 . It can be concluded that the calibration procedure of the capacitance meter and the measuring procedure of the liquid fraction assure the reliability of the proposed technique.

The images reconstructed from the capacitance measurements, using the linear back projection algorithm with a cut-off level resource, showed a good agreement with the real phase distribution inside the sensor, validating the developed methodology.

\section{REFERENCES}

Azevedo Filho, A. C., 2002, Desenvolvimento de um sistema de tomografia capacitiva para processos multifásicos. PhD. Thesis, Faculdade de Engenharia Mecânica, Universidade Estadual de Campinas - UNICAMP.

Bottcher, C. J. F., 1952, Theory of electric polarization. New York: Elsevier.

Bracewell, R. H., and Riddle, A. C., 1967, Inversion of fan beam scans in radio astronomy, Astrophysics Journal, No. 150, pp. 427-434.

Geldart, D., and Kelsley, J. R., 1972, The use of capacitance probes in gas fluidized beds, Powder Technol. Vol. 6, pp. 45-50.

Hage, B., and Werther, J., 1997, The guarded capacitance probe - a tool for the measurement of solids flow patterns in laboratory industrial fluidized bed combustors, Powder Technol., No. 93, pp. 235245.

Huang, S. M., Xie, C. G., Vasina, J., Lenn, C., Zhang, B. F., and Beck, M. S., 1992, Experimental evaluation of capacitance tomographic flow imaging systems using physical models, Proc. European concerted Action on Process Tomography, pp. 295308.

Kak, A. C., and Slaney, M., 1988, Principles of computerized tomographic imaging, New York: IEEE.

McKee, S. L., Williams R. A., Dyakowski, T., and Bell, T. A., 1993, Industrial applications of electrical tomography to conveying processes, Proc. I Chem. E Res. Event, Birmingham, 6-7 January, Inst. Chem., Eng., pp. 726-728.

Jing, L., Shi, L., Zhihong, L., and Meng, S., 2009, An image reconstruction algorithm based on the extended Tikhonov regularization method for electrical capacitance tomography, Institute of Engineering Thermophysics, Chinese Academy of Sciences, Vol. 42, pp. 368-376.

Marioli, D., Sardini, E., and Taroni, A., 1993, High-accuracy measurement techniques for capacitance transducers, Meas. Sci. Technol. Vol. 4, pp. 337-343.

Meredith, R. E., and Tobias, C. W., 1960, Resistance to potential flow through a cubical array of spheres, J. Appl. Phys., No.. 31, pp. 1270 - 1273.

Morse, T. D., and Bellou, C. D., 1951, The uniformity of fluidization, its measurement and use". Chem. Engng. Prog., No. 47, pp. 199-211.

Nooralahiyan, A.Y., Hoyle, B.S. and Bailey, N.J., 1993, Application of a neural network in image reconstruction for capacitance topography, Proc. European Concerted Action on Process Topography/UMIST, pp. 144-147.

Ramnchandran, G., and Lakashinarayanan, A. V., 1971, Three dimensional reconstructions from radiographs and electron micrographs: Application of convolution instead of Fourier transforms, Proceedings of the National Academy of sciences, Vol. 68, pp. 2236-2240.

Rayleigh, J. W. S. B., 1892, On the influence of obstacles in rectangular order upon the properties of a medium, Philos. Mag., No. 34, pp. 481.

Xiangyuan D., 2009, Fast Image Reconstruction Algorithm for Electrical Capacitance Tomography, 4th IEEE Conference on Industrial Electronics and Applications, pp. 3753-3756.

Xie, C. D., Plaskowski, A., and Beck, M. S., 1989, 8-Electrode capacitance system for twocomponent flow identification. Part 2: flow regime identification, IEE Proceedings-G, Vol. 136, No. 4, pp. 184-190.

Xie, C. G. et al., 1993, Review of process topography image reconstruction methods in K.T.V. Grattan and A.T, Augousti (eds). Sensors VI: Technology, Systems and Applications. Bristol: Institute of Physics, pp. 341-346.

Yang, W. Q., and York, T. A., 1999, New ACbased capacitance tomography system, IEE Proc Sci. Meas. Technol. Vol. 146, No. 1, pp. 47-53.

Yang, W. Q., 2010, Design of electrical Capacitance Tomography Sensors, Meas. Sci. Technol., Vol. 21, pp. 1-13.

Received: June 07, 2009

Revised: July 07, 2009

Accepted: August 07, 2009 